\title{
ENTRE REBUSQUES, CHANGAS Y OFICIOS. MODOS DE GANARSE LA VIDA DE JÓVENES QUE PARTICIPAN EN PROGRAMAS DE FORMACIÓN SOCIOLABORAL EN CONTEXTOS DE POBREZA URBANA DE LA CIUDAD DE ROSARIO ${ }^{1}$, ARGENTINA
}

\author{
SOFÍA VITALI (ISHIR-CONICET)2
}

\begin{abstract}
RESUMEN
En este artículo se analizan los modos de ganarse la vida de jóvenes que participan de dispositivos estatales orientados a la inclusión laboral en contextos de pobreza urbana de la ciudad de Rosario, Argentina. El foco puesto en el universo de posibilidades, relaciones y expectativas que configuran la reproducción de la vida de los y las jóvenes permite comprender tensiones, resignificaciones y adaptaciones de la gestión cotidiana de estas políticas. Particularmente se destaca cómo se incorporan prácticas y dinámicas locales que son puestas en juego en las interacciones cotidianas entre trabajadores estatales y jóvenes.
\end{abstract}

PALABRAS CLAVES: ESTRATEGIAS DE REPRODUCCIÓN DE LA VIDA, POLÍTICAS JUVENILES SOCIOLABOLARES, POBREZA URBANA

\footnotetext{
${ }^{1}$ Rosario se encuentra ubicada en la Provincia de Santa Fe (Argentina), sobre el margen oeste del río Paraná; posee un total de 948.312 habitantes según datos del último Censo Nacional, realizado en el año 2010. Actualmente ocupa el tercer lugar entre las ciudades más pobladas del país, con una densidad de $5.089 \mathrm{hab} / \mathrm{km}$, representando el $40 \%$ de la población provincial. Su población es básicamente urbana (99\%), con un $52,5 \%$ de mujeres y un $47,5 \%$ de hombres.

2 Doctora en Humanidades y Artes (mención Antropología) y licenciada en Antropología por la Facultad de Humanidades y Artes de la Universidad Nacional de Rosario (UNR). Becaria posdoctoral del Consejo Nacional de Investigaciones Científicas y Técnicas (CONICET) en el Instituto de Investigaciones Socio-históricas Regionales (ISHIR). Correo electrónico: sofiamvitali@gmail.com
} 


\title{
ENTRE REBUSQUES, CHANGAS E OFÍCIOS. FORMAS DE GANHAR A VIDA DOS JOVENS QUE PARTICIPAM DE PROGRAMAS DE FORMAÇÃO SOCIOLABORAL EM CONTEXTOS DE POBREZA URBANA NA CIDADE DE RosÁrio, Argentina
}

\begin{abstract}
RESUMO
Este artigo analisa as formas de ganhar a vida dos jovens que participam dos dispositivos estatais orientados à inclusão laboral em contextos de pobreza urbana na cidade de Rosário, Argentina. O foco está no universo de possibilidades, relações e expectativas que configuram a reprodução da vida dos e das jovens e permite compreender tensões, ressignificações e adaptações da gestão cotidiana destas políticas. Em particular, é destacada a forma como as práticas e dinâmicas locais são incorporadas e postas em prática nas interações cotidianas entre os trabalhadores do Estado e os jovens.

PALAVRAS-CHAVE: FORMAS DE GANHAR A VIDA, POLÍTICAS SOCIOLABORAIS PARAA JUVENTUDE, POBREZA URBANA

\section{AMONG REBUSQUES, CHANGAS AND OFICIOS. WAYS TO MAKE A LIVING OF POOR URBAN YOUNG PEOPLE PARTICIPATING IN LABOR INCLUSION POLICIES IN ROSARIO, ARGENTINA}

\begin{abstract}
This article analyzes the ways to make a living of young people participating in state labor inclusion programs in contexts of urban poverty in Rosario, Argentina. The focus on the universe of possibilities, relationships, and expectations that shape the reproduction of the lives of male and female young people allows understanding tensions, resignifications, and adaptations of the daily management of these policies. Particularly, it is highlighted how local practices and dynamics are incorporated into daily interactions between state workers and youth.
\end{abstract}

KEYWORDS: WAYS TO MAKE A LIVING, LABOR INCLUSION POLICIES FOR YOUNG PEOPLE, URBAN POVERTY 


\section{INTRODUCCIÓN}

En los últimos años, el campo de estudio vinculado al desarrollo de políticas de inclusión sociolaboral para jóvenes ha ganado terreno al interior de la agenda académica. Su auge y despliegue coincide, de alguna manera, con la percepción del momento crítico que atravesó la población juvenil en los inicios de la era neoliberal y su emergencia como «cuestión social» al interior de las políticas públicas y sociales. Esto se debió a los profundos cambios en el mundo laboral y de los quiebres en el vínculo entre educación y empleo, que repercutieron diferencialmente para la población joven (Navarrete, Padrón Innamorato y Silva Arias, 2012; Jacinto, 2002; Sánchez, 2006).

Entre las indagaciones del campo se destaca que la mayoría de los programas de inserción laboral juvenil comienzan a implementarse desde fines de la década del 80 e inicios de la década del 90, en el marco de procesos de focalización de la política social a grupos particulares (Balardini, 1999, Krauskopf, 2004). Estas iniciativas estatales recuperaron en su formulación la teoría del capital humano a partir de la cual se otorga centralidad al fomento de las competencias y aptitudes de las personas para poder aumentar su empleabilidad y poder vender su fuerza de trabajo, reivindicando así el papel de la educación en el desarrollo económico y la estructuración del empleo (Brown; 2017; Shapiro, 2000; De Ibarrola, 2002). Bajo estas prerrogativas, la mayoría de los dispositivos estatales se implementaron de manera focalizada a los y las jóvenes que no habían concluido la escuela secundaria y/o vivían en contextos de pobreza, mediante componentes de capacitación, intermediación para la inclusión de los jóvenes en empleos y autoempleos y transferencias monetarias 
condicionadas (PTMC) ${ }^{3}$ (Jacinto, 2010; Navarrete, Padrón Innamorato y Silva Arias, 2012; Valverde Viesca, 2012).

En Argentina, a partir de la posconvertibilidad ${ }^{4}$, se inicia una etapa de recuperación económica que sin embargo no logró revertir el deterioro estructural del mercado de trabajo que habían tenido a los jóvenes entre uno de los grupos particularmente afectados (Pérez, 2010; Castagna y Véntola, 2015). En este contexto, diferentes estudios identificaron las persistencias y nuevas problemáticas vinculadas al empleo juvenil, entre ellos su sobrerrepresentación en los niveles de precariedad laboral (tanto en el sector formal como informal) y la rotación de las ocupaciones juveniles (Muñiz Terra, Roberti, Deleo y Hasicic, 2013). En lo que respecta a la intervención del Estado hacia este sector social, se destacan ciertos cambios vinculados al denominado «giro productivo» de la política social, a partir del cual se sostendrá el sesgo focalizador y promotor del «capital humano», pero se pasará de una política orientada a la capacitación laboral a la generación de las «disposiciones» hacia el empleo (Jacinto, 2008; Pérez, 2013; Cortes y Kesller, 2013; Assusa, 2013; Isadovich, 2016). Por otro

${ }^{3}$ Los denominados Programas de Transferencias Monetarias Condicionadas (PTMC) consisten en la entrega de recursos monetarios y no monetarios a familias o personas en situación de vulnerabilidad con la condición de que los perceptores cumplan con ciertas conductas asociadas al aumento del capital humano y a la mejora de la empleabilidad para que puedan (luego) ingresar a algún trabajo lucrativo o para mejorar la calidad de sus inserciones laborales. Al interior de la literatura académica, estas iniciativas también son denominadas como «políticas activas de empleo», en tanto son dispositivos que otorgan ingresos a las personas en situación de desempleo y que exigen a cambio contraprestaciones vinculadas a la realización de tareas laborales o a participación en actividades de capacitación y formación que aumenten su empleabilidad (Brown, 2017).

${ }^{4}$ Las transformaciones que se generaron a partir de 2003 en Argentina están atravesadas por el período iniciado durante la postconvertibilidad, a partir de la cual se generaron ciertos contrastes con respecto al desenvolvimiento económico, social y político que caracterizó a los años noventa. Dichos contrastes se desplegaron tanto en lo que respecta a la recomposición de la acumulación capitalista como en la dominación social, posterior al estallido de la crisis en el año 2001 (Marticorena, 2014). Entre las acciones desarrolladas por el Estado se resalta un conjunto decisiones económicas sobre el modelo de crecimiento, el aumento de su capacidad regulatoria en la inspección del trabajo y el fortalecimiento de la negociación colectiva (Salvia, 2010). 

de jóvenes que participan en programas de formación sociolaboral en contextos de pobreza urbana de la ciudad de Rosario, Argentina

lado, se destaca que muchos de estos programas se orientaron a atender la demanda social organizada a través de movimientos y actores de la sociedad civil (principalmente sindicatos, ONG y organizaciones de desocupados), con una identificación más territorial que sectorial, que recibieron apoyo para la generación de empleo en el marco de emprendimientos sociales cooperativos (Jacinto, 2016).

Más allá de estas innovaciones mencionadas, las iniciativas estatales presentaron particulares dificultades en la integración laboral de la población juvenil pobre (Millenar, 2012). Esto se debe tanto a las características fragmentarias y de corta duración de los dispositivos estales, como por las escasas posibilidades con que cuentan los y las jóvenes, bajo las particulares condiciones familiares y socioeconómicas en las que viven (Isadovich, 2016; Vitali, 2018). Estas dificultades se vieron incrementadas al calor de las transformaciones en los dispositivos estatales sufridos a partir de $2015^{5}$, momento en el cual se reactualizan discursos estatales vinculados a lógicas meritocráticas y del merecimiento, tanto al interior de las componentes de capacitación (profesional y/o de oficio), como para el acceso de los y las jóvenes a subsidios $\mathrm{y} / \mathrm{o}$ microcréditos. Desde estas nuevas prerrogativas, estudios destacan que se tendió a una mayor culpabilización de la situación de exclusión a los propios destinatarios, en tanto el «éxito» o «fracaso» de su posterior inserción laboral es leído en términos de la propia capacidad, esfuerzo y desempeño individual de los mismos (Vázquez, 2019).

En este trabajo nos proponemos contribuir a este campo de estudio, atendiendo no solo a los procesos de implementación de estas políticas en el

${ }^{5}$ En el año 2015 , a partir de la victoria electoral de la coalición política «Alianza Cambiemos» para la presidencia, se produce un ascenso de sectores de la derecha al poder político argentino, con un relanzamiento de iniciativas neoliberales. Bajo esta nueva gestión de gobierno se produjeron una serie de modificaciones de la orientación política y económica del país que gravitaron en las políticas de inclusión laboral juvenil. 
ámbito local/barrial, sino también restituyendo los heterogéneos modos de ganarse la vida de los y las jóvenes y cómo estos se interpenetran en la gestión cotidiana del trabajo de un programa estatal. Para su abordaje, recuperamos la categoría de modos de «ganarse la vida», la cual permite ampliar el análisis de las relaciones laborales «de dependencia» o «empleo» para atender a la diversidad de prácticas puestas en juego para lograr la subsistencia (Narotzky, 2013; Narotzky y Besnier, 2014; Fernández Álvarez, 2016). Esto nos permitirá bucear por los espacios formales, informales y domésticos a los que acceden los y las jóvenes y que permean la gestión cotidiana del trabajo, desbordando los límites impuestos desde las disposiciones formales de las políticas.

Metodológicamente, recuperamos el enfoque antropológico, el cual nos permite rescatar los sentidos, valores, prácticas y significaciones que construyen los distintos sujetos sociales significativos de nuestra problemática (Menéndez, 2010). En cuanto al recorte empírico de la investigación incluimos al Programa «Nueva Oportunidad ${ }^{6}$ de la provincia de Santa Fe, el cual tiene como población objetivo a jóvenes de entre 14 y 30 años que habiten en diferentes asentamientos irregulares de la ciudad. Nuestro trabajo de campo se realizó en dos de las dependencias estatales descentralizadas en que este se implementa: el Centro Cultural «El Obrador»y el Centro de Convivencia Barrial Flammarion, ambos localizados en asentamientos informales, uno en el distrito Oeste de la ciudad Barrio Toba- y otro, en el distrito Sudoeste -Flammarión-. Entre los diferentes actores con quienes sostuvimos entrevistas y observaciones participantes ubicamos a los funcionarios estatales implicados en el diseño normativo del Programa, los coordinadores y trabajadores estatales involucrados

\footnotetext{
${ }^{6}$ Este programa pasó a denominarse «Programa Santa Fe Más» en el año 2019, debido al recambio de gestión de gobierno de la provincia de Santa Fe. Es importante mencionar que el cambio de gestión no implicó modificaciones en sus principales líneas de trabajo.
} 

de jóvenes que participan en programas de formación sociolaboral en contextos de pobreza urbana de la ciudad de Rosario, Argentina

en la implementación y a los jóvenes que participan de los dispositivos seleccionados.

La discusión que aborda el texto recupera en primer lugar, los lineamientos y modalidades de la intervención de las políticas de inclusión laboral para jóvenes; en segundo lugar, un recorrido por los modos de ganarse la vida de los y las jóvenes que participan de los dispositivos estatales y, finalmente, las relaciones laborales desplegadas en las interacciones cotidianas entre los trabajadores estatales y los destinatarios.

\section{Políticas de INSERCIÓN LABORAL PARA JÓVENES: DIAGNÓSTICO, SUPUESTOS Y ACCIONES}

En diciembre del año 2013, el gobierno de la provincia de Santa Fe puso en marcha el Programa «Nueva Oportunidad», en el marco de la percepción del «riesgo»y «peligrosidad» que afronta la población joven en la ciudad de Rosario, producto del aumento de la violencia ${ }^{7}$ en contextos de vulnerabilidad social y urbana (Informe ABRE). Este se propone promover la «inclusión sociolaboral» de sus destinatarios, a partir de generar trayectos de capacitación y formación laboral en diferentes oficios y rubros de actividad. Por otro lado, relevamos que se abordan diferentes problemáticas que trascienden lo laboral, como es el consumo problemático, violencia de género, entre otras.

En términos formales, la propuesta consiste en el dictado de cursos de capacitación laboral de seis meses de duración, que puede ser extendida en el

\footnotetext{
${ }^{7}$ Según informes estatales entre el 2011 y 2012 se produjo un incremento del 29,3\% de los homicidios dolosos respecto al año 2010 (Dirección de Análisis CriminalMinisterio de Seguridad-Unidad Regional II de Policía). Esta escalada de violencia se generó - en parte - por disputas territoriales entre distintas «bandas» asociadas al narcotráfico (Juan Murray, Secretario de la Procuraduría de Narcocriminalidad (Procunar), La Nación, 11 de abril de 2014).
} 
tiempo para dar continuidad al proceso formativo de los jóvenes. Esto último se produce mediante su línea de trabajo «Nexo Oportunidad», que prevé la conformación de unidades productivas (emprendimientos) y/o la inserción de los jóvenes en pasantías en empresas. Ambos trayectos contemplan una beca estímulo mensual para que puedan complementar con la asistencia y aprobación de las prestaciones disponibles.

Es importante destacar que desde el Programa se otorga centralidad a la «proximidad territorial» con la población destinataria y, por lo tanto, los equipos de trabajo se localizan en las instituciones estatales de los contextos sociourbanos donde habitan los jóvenes (Ordenanza Municipal: Programa Nueva Oportunidad). Para su implementación, el gobierno provincial y municipal realizó convenios con instituciones de formación y capacitación laboral, organizaciones sociales y dependencias estatales descentralizadas que se localizan al interior de los barrios donde habitan los jóvenes. De esta manera, los equipos de trabajo integran a capacitadores en diferentes rubros productivos, referentes sociales y trabajadores estatales territoriales. Estos equipos se sostienen sobre una planta de trabajadores sumamente precarizada bajo diversas modalidades de relación laboral entre las que identificamos contratos a través de ONG, contratos transitorios, contratos de locación de servicios monotributistas, pasantías, etc.

En cuanto al diagnóstico de la problemática laboral juvenil, identificamos que este programa, al igual que los desplegados a nivel nacional, caracteriza a los jóvenes como carentes de «cultura del trabajo»y, por tanto, «desafiliados» de las instituciones laborales (Assusa, 2014). Bajo esta concepción, los funcionarios del programa destacan que los jóvenes carecen de experiencias laborales y educativas $\mathrm{y}$, de esta manera, son pensados en términos de sus dificultades «subjetivas»y «materiales» para vincularse con el trabajo y/o para conformar emprendimientos productivos. Como relata una de las trabajadoras en territorio: 
Muchos [jóvenes] están muy lejos en su realidad con lo que tiene que ver con el trabajo, con lo que eso implica, con un ingreso económico digno, derechos, jornada de trabajo y a largo plazo. Porque algunos tienen noción de jornada de trabajo por changas $^{8}$ que han realizado sus padres, ellos no tanto, algunos poquitos trabajaron en alguna obra de construcción. Los trabajos que existen para los chicos no llegan ni siquiera a la changa (...). La cuestión de reglamentar o introducir nociones que tienen que ver con posibilidades que se le pueden llegar a presentar para un trabajo futuro o de pedido de venta o demás donde necesiten. Pero no sé cuándo eso podrá tener lugar en el pensamiento de los chicos, porque con este grupo estamos en una etapa muy primaria de lo que puede ser un emprendimiento. (Sabina, acompañante del Programa en Barrio Toba, 27 de julio de 2016)

En el testimonio emerge, en primer lugar, la caracterización sobre la lejanía de los participantes con el trabajo — que podemos definir como «formal»-y, por lo tanto, con las rutinas y normas asociadas a este, entre las que Sabina menciona, la jornada de trabajo, los derechos laborales y la estabilidad o el «largo plazo». En segundo lugar, aparecen elementos a los que se orientan las intervenciones, tales como «reglamentar o introducir nociones» que permitan el ingreso al trabajo remunerado o la conformación de emprendimientos.

En paralelo a esto, la retórica del programa apunta a generar un abordaje «integral» de la problemática, la cual se vincula con que, además de la orientación y capacitación laboral, los equipos territoriales generen lazos de cercanía y confianza con los y las jóvenes, realicen actividades y estrategias de contención

\footnotetext{
${ }^{8}$ Changas: acepción argentina utilizada para referirse al trabajo ocasional, generalmente en tareas menores, realizado de manera informal y sin ningún tipo de protección, que permite generar ingresos irregulares para la subsistencia, por ejemplo trabajos de albañilería, ventas callejeras: «hacer una changa».
} 
individual y grupal y que les permitan «pensar un proyecto de vida». En palabras del Director del Programa:

La finalidad de los equipos es acompañar procesos colectivos e individuales de manera integral, que permita la revisión de sus estrategias de vida (...) trabajar no solamente lo que tiene que ver con el curso y la posibilidad después, de un empleo o de un trabajo a partir de una formación; sino de generar un vínculo y que los jóvenes en función de ello puedan pensar un proyecto de vida (...) De esta manera, su finalidad trasciende el aprendizaje del oficio, orientándose a la generación de espacios de encuentro, participación e intercambio entre sus participantes. (Luciano Vigoni, Director Provincial del Programa, diario El Ciudadano, 2 de noviembre de 2018)

Como se destaca en el relato, entre las disposiciones y orientaciones expuestas por el directivo del Programa, se busca la generación de estrategias en territorio que permitan un abordaje «integral» de sus problemáticas y posibiliten generar «cambios subjetivos» en los y las jóvenes. Este tipo de abordaje fue problematizada en diferentes investigaciones (Pérez, 2013; Jacinto, 2010), desde las cuales se indica que este tipo de concepciones están permeadas por una interpretación «voluntarista», en tanto reducen el problema de la inserción laboral de estos conjuntos sociales, a una serie de características «subjetivas» y «competencias» de los sujetos. Advierten, igualmente, que el problema de esta perspectiva es no incluir en su diagnóstico las propias características del mercado laboral y sus posibilidades de acceso, responsabilizando así al propio sujeto trabajador de la situación de desempleo. Además de estas cuestiones, nos parece que las mismas tienden a desconocer las propias estrategias y dinámicas laborales que despliegan los jóvenes y que nos interesa profundizar en el próximo apartado. 

de jóvenes que participan en programas de formación sociolaboral en contextos de pobreza urbana de la ciudad de Rosario, Argentina

\section{TRABAJOS DE VERDAD, REBUSQUES Y AYUDAS.}

\section{LOS UNIVERSOS DE POSIBILIDAD, RELACIONES Y EXPECTATIVAS DE TRABAJO DE LOS Y LAS JÓVENES EN CONTEXTOS DE POBREZA URBANA}

En nuestra llegada a los barrios Toba y Flammarion, lo primero que capturó nuestra atención era cómo su fisonomía se encontraba completamente invadida por puestos callejeros para la venta de alimentos o reventa de artículos (ferias populares) o «quioscos» localizados en domicilios particulares. No resultaba menor la cantidad de trabajadores que circulaban en bicicletas con sus uniformes o ropa de trabajo durante los horarios de arribo a sus hogares. Este panorama que afloraba en la mera observación de nuestro estar allí nos reclamaba analizar cómo se configuraban los universos de posibilidad, relaciones y expectativas laborales de los y las jóvenes que asistían a los dispositivos estatales orientados a la «inclusión sociolaboral».

A partir de esto, en términos conceptuales, nos resultó imprescindible partir de una categoría amplia de trabajo, que nos permitiera abordar las heterogéneas formas concretas en las que se desarrolla y la variedad de relaciones económicas, sociales y culturales que lo constituyen (Menéndez, 1990). Para su abordaje, entonces, la conceptualización de modos de ganarse la vida, nos permitió captar la mayor porosidad, pluralidad y diversificación de las relaciones capital-trabajo y su ampliación y articulación con espacios familiares y vecinales. Asimismo, para comprender los sentidos y valoraciones vinculados a dichas actividades se atendió a cómo opera la transmisión y circulación de sentidos mediatizadas en las relaciones intergeneracionales producidas al interior de la familia. Esto último tiene particular importancia, en tanto resultaba frecuente que los y las jóvenes nos mencionaran que eran sus padres o personas a cargo quienes les inculcaban o exigían «hacer algo» en relación con una actividad laboral y/o formativa que les permitiera aprender un oficio y acceder a un trabajo, con el cual 
realizar un aporte económico que ayudara a la familia o para solventar sus propias necesidades. Por otro lado, identificamos que las concepciones y prácticas vinculadas al trabajo son transmitidas generacionalmente desde su temprana edad, las cuales inciden en las trayectorias laborales y en la construcción de ciertas desigualdades de género alrededor del trabajo.

Entre los y las jóvenes entrevistados hemos registrado que el acceso al trabajo asalariado suele ser más frecuente entre los varones, quienes tienden a emplearse por salarios muy bajos, con largas jornadas laborales y en empleos de gran demanda física. En el caso de las mujeres, estas tienen escasas posibilidades de asalarización y presentan mayores trayectorias en trabajos de circuitos informales, cuentapropia comercial y sobrecarga de trabajo doméstico y de cuidado.

Los sectores en los que se insertan los varones son principalmente la construcción y en menor medida la metalmecánica y manufacturera. También relevamos inserciones dentro del sector servicio, como empresas de limpieza, gastronomía y hotelería, comercio. Dentro de las posibilidades de inserción de las mujeres identificamos que los sectores más frecuentes son dentro del sector servicio en la rama del comercio, la gastronomía, servicio doméstico, hotelería. También identificamos recorridos al interior del sector textil.

Entre las estrategias para el ingreso a los trabajos remunerados hemos relevado la importancia de los lazos familiares y conocidos, principalmente en el sector de la construcción. Los jóvenes nos relataban que familiares y vecinos solían consultarlos si les interesaba trabajar de manera temporal por algún trabajo puntual, en el que se requería la disponibilidad de una mano de obra fluctuante y barata. Así, los adultos del barrio solían reclutarlos para trabajos puntuales en diferentes sectores: como la construcción (aprendices) y la gastronomía (cadetería, mozos, lava copas, ayudantes de cocina). De esta manera, podemos afirmar que el acceso al trabajo remunerado se da generalmente bajo «redes de 
recomendabilidad» entre conocidos y parientes. Es importante destacar que estas redes no solo operan como mecanismo para el ingreso, sino que también como mecanismo disciplinante al interior del ámbito laboral. Con esto nos referimos a lo que los jóvenes nos mencionaban, que debían «hacer quedar bien» o «no armar bardo», ya que esto podría perjudicar a quienes los recomendaban y romper los propios lazos entre los conocidos.

En líneas generales, las inserciones dentro de estos empleos presentan altos niveles de no registro y carencia de derechos laborales, como también empleo registrado pero bajo contratos de corta duración a través de pasantías y/o períodos de prueba. Por otro lado, registramos que suelen realizar tareas no calificadas, empleándose como aprendices, peones, etc. Sin embargo, durante el trabajo de campo, observábamos que en los relatos de los y las jóvenes, esta forma fluctuante de utilización de la fuerza de trabajo, caracterizada por su flexibilidad contractual e inestabilidad, aparecía en los sentidos completamente naturalizada y, por lo tanto, recubierta de cierta legitimidad. Asimismo, una cuestión que nos llamó la atención es cómo el ingreso a este tipo de empleos más allá de la precariedad descrita— es significada como «verdaderos» trabajos.

Como rememora Lucas —un joven del barrio Flammarion que por primera vez ingresa, mediante una pasantía de una duración de seis meses, a una fábrica de la industria gráfica-, más allá de lo limitado y precario de su ingreso, este es significado como su «primer trabajo de verdad».

-Yo no estoy trabajando ni en una fábrica ni en blanco, yo solamente estuve en la etapa de trabajo, empecé a trabajar desde los 14 años; mi vieja era la que me jodía, la que me decía «tenés que tener un laburo» porque era yo el más grande — mi hermana ya se había ido - y quedaba yo con tres pibes, tres hermanitos: de dos la más chiquita y el otro que ya es grande, ahora tiene 16 años; entonces yo agarré y (...) empecé a salir en la calle. Salí a la calle y les preguntaba a las señoras si no quería que les tire la basura o, 
de último, si no querés que te tire las ramas que tenés ahí afuera en la vereda, escombros, te entro la arena, cualquier cosa.

— ¿Todas cosas acá en el barrio, digamos?

-Claro, claro; cosas de changas, y bueno, así fueron surgiendo trabajos: ayudante de albañil ayudante de herrería... (...) Mi primer trabajo de verdad es el de Borsalino Express [una compañía de industria gráfica], me van a poner en blanco, voy a tener tarjeta para cobrar y todo. (Lucas, joven de 19 años, barrio Flammarion, noviembre de 2017)

Como se expresa en el relato, dentro de la variedad de actividades laborales realizadas, el trabajo de «verdad» está asociado a la condición asalariada, bajo relación de dependencia y, en este caso particular, con la posibilidad de estar en «blanco», es decir, adquirir los derechos correspondientes del registro como empleado, con excepción de la estabilidad laboral, ya que su ingreso se da en el marco de una pasantía. De esta manera, podemos plantear que se establecen ciertas «jerarquías» del trabajo, donde la forma asalariada adquiere mayor «valor» frente a otras opciones laborales. Esto también fue documentado en la investigación de Silvana Sánchez (2006) en el barrio Toba, donde destaca que entre los jóvenes, más allá de su escasez y condiciones del trabajo asalariado, este sigue ocupando un lugar central en tanto "se lo habla, se lo busca, se lo espera, se despliegan esfuerzos y estrategias para tratar de conseguirlo" (Sánchez, 2006: 118).

Asimismo, relevamos que, más allá del «valor» que adquiere el trabajo asalariado, la estadía y duración de los jóvenes en estos trabajos varía en función de cuánto «aguantaban» los ritmos de explotación y las escasas remuneraciones a los que se ven expuestos. En tal sentido, resultaba frecuente que más allá de los deseos de tener un trabajo «de verdad», muchos jóvenes nos comenten que preferían abandonar el empleo y buscar otras estrategias de generación de recursos frente a los altos niveles de explotación a los que se ven expuestos. Como nos cuenta Gonzalo, un joven del barrio Toba, el trabajo pesado y 
monótono que realizaba para una carbonería cercana al asentamiento es motivo de su deserción laboral:

-Sí, yo trabajé en una carbonería, queda cerca de los FONAVI, ahí estuve poco porque no me gustaba.

— ¿Por qué, cómo es ese trabajo?

- Y es muy pesado ese trabajo, tenés que cargar camiones y armar las bolsas de carbón, tenés que trabajar con pala, con la pala cargar, meter en bolsas y después llenar el camión.

- ¿Y eran muchas horas?

- Sí, mucho, desde la mañana hasta la tarde, entraba a las 8 y a veces salía a las 17 o 19 horas, según cuánto trabajo haya. Igual trabajé poco ahí, aguanté tres meses. (Gonzalo, joven de 19 años, barrio Toba, noviembre de 2017)

Como se destaca en el relato, las condiciones de empleo, las cuales son mal remuneradas, extenuantes, no calificadas ni gratificantes, se vuelven intolerables, razón por la cual decide abandonar el trabajo. De esta manera, observamos que la incorporación de la disciplina laboral es un proceso de múltiples tensiones y resistencias que se vuelve particularmente problemático para los jóvenes. Como documentan diferentes antecedentes, las condiciones de precariedad laboral en la que se insertan son la principal fuente de «reticencia» al trabajo de los jóvenes de sectores populares (Sánchez, 2006; Jacinto, 2010; Bourgois, 2010).

Ahora bien, muchas de las actividades y tareas orientadas a la generación de ingresos y/o condiciones de reproducción y consumo que realizan los y las jóvenes se desarrollan por fuera de las relaciones laborales asalariadas. Estas se desenvuelven en ámbitos heterogéneos, entre los que se incluyen el espacio público y el hogar y contienen un conjunto amplio de relaciones y lazos familiares y vecinales, así como vínculos personales con referentes de organizaciones sociales del territorio y con trabajadores estatales. Estas prácticas 
suelen estar asociadas al «rebusque»y son percibidas de manera desigual a los trabajos de «verdad».

Identificamos que estas actividades suelen ser sobrellevadas en mayor proporción por las jóvenes debido a las inequidades de género presentes en el mercado de trabajo, el cual muestra una menor oferta de empleos y brechas salariales desfavorables para las mujeres. También responde a ciertos mandatos de género que confinan a estas últimas a labores al interior del hogar y del barrio.

Entre estas actividades se incluye la participación en circuitos comerciales informales, como la reventa de indumentaria y accesorios, ropa usada a partir de la instalación de «roperitos» en ferias populares localizadas al interior de los barrios o en sus adyacencias, la venta ambulante en puestos callejeros o por catálogo dentro del barrio. La participación de los y las jóvenes en estas actividades puede ser para «ayudar» a sus progenitores o para generar ingresos para su propia familia, en los casos en los que ya tengan hijos. En tal sentido, relevamos que estas prácticas son sobrellevadas por diferentes integrantes de las unidades domésticas, generalmente por las mujeres ayudadas de sus hijos/as mayores.

A continuación reconstruimos el relato de Rita, una joven del barrio Toba, quien comenzó con la reventa de ropa en ferias populares a los 14 años de edad de la mano de su madre. Ella comenta que en ese entonces, su madre inicia la actividad para «juntar plata» para la celebración de sus quince y que desde entonces «no dejaron más». Ambas viajan semanalmente a «La Salada», una de las ferias populares más grandes de América Latina, localizada en Buenos Aires, donde consiguen ropa a muy bajo costo que luego revenden en las ferias del barrio y en sus domicilios. Rita nos comenta que comenzó a vender por su cuenta cuando queda embarazada de su primer hijo, para poder «conciliar» la vida laboral y del hogar. En palabras de Rita:

—Y cómo fue que empezaste a vender en la feria vos sola? 
- Yo vendía con mi mamá y después cuando tuve a Benja [su hijo] empecé a vender para mí, me gustó porque, digo, bueno, la feria me queda cerca, no tengo que ir todos los días y si podemos trabajar en eso está rebueno; yo, por Benjamín, más que nada (...). Porque mi marido trabaja y yo así puedo generar un ingreso y cuidar al nene. (Rita, joven de 22 años, barrio Toba, 21 de diciembre de 2017).

Esta situación resultó significativa en las unidades domésticas de primer ciclo de vida, es decir, aquellas que tienen hijos pequeños, donde las jóvenes frecuentemente aludían a la imposibilidad de trabajar fuera del hogar por la necesidad de atender a sus hijos. En los relatos se remarcaba la importancia del proyecto de la maternidad, las responsabilidades y obligaciones en el cuidado de sus hijos, que recaían generalmente sobre ellas.

También encontramos que los y las jóvenes participan en diferentes organizaciones sociales y políticas del territorio como parte de sus posibilidades de generar ingresos dinerarios. Entre ellas registramos que algunos jóvenes reciben «becas» de programas estatales o «ayudas económicas» como contraprestación de actividades comunitarias coordinadas por organizaciones y movimientos sociales como comedores y copas de leche, huertas comunitarias, emprendimientos de productos y cooperativas de trabajo vinculadas a actividades de mantenimiento del barrio. Estas últimas suelen estar gestionadas por organizaciones sociales del territorio que establecen contratos de prestación de servicios para el Estado municipal. Como nos relata Mariano, un joven de 18 años:

-Bueno, yo trabajé en una cooperativa de mantenimiento que hay acá en el barrio, estuve trabajando ahí como dos años, pero después no fui más, dejé de ir por tres meses y no me dejaron volver más.

— ¿Cómo fue que empezaste ahí?

- Yo arranqué porque me dijo mi hermana, yo en ese momento estaba al pedo, sin hacer nada, y entonces mi hermana me empezó a decir para que vaya. Eso lo maneja Tata, un vecino del barrio, y en ese momento había lugar y me anoté, se trabaja por mes ahí. 
— ¿Cómo es el trabajo?

- Se arman cuadrillas de quince personas y nos dividimos las cuadras del barrio. Hacemos zanjeo y limpieza, es un trabajo pesado cuando hay que destapar las zanjas, el trabajo con pala y camión juntando todo. Nos hacen comenzar a las 8 de la mañana hasta el mediodía, más o menos, y nos pagan tres mil pesos. Ahí también trabaja mi hermana. (Mariano, joven de 18 años, barrio Toba, 13 de noviembre de 2017).

En el relato se destaca cómo los vínculos entre conocidos, familiares y referentes del barrio son fundamentales para el reclutamiento de trabajadores para estas cooperativas. Asimismo, identificamos que la organización del trabajo está regida por pautas de cumplimiento de horarios, asistencia y dividida por grupos del trabajo. Como ha sido documentado en las investigaciones realizadas por Virginia Manzano "trabajar con planes sociales", recrea modalidades y pautas del mundo laboral, como la organización de turnos de trabajo (mañana/tarde), el registro de asistencia e inasistencias, la justificación del ausentismo y los períodos de vacaciones anuales (Manzano, 2013: 198).

Hemos registrado que estas actividades son significadas como posibilidad de «rebusque», en tanto se constituye como una forma de ganarse la vida que les permite generar ingresos bajo diferentes formas, adquiriendo gran importancia para su sostenimiento las redes familiares, vecinales y con organizaciones sociales.

Finalmente, hemos registrado una serie de actividades, entre las que se incluyen tareas de limpieza, elaboración de la comida y cuidado de hermanos/as menores, las cuales son significadas como «ayuda» y son sobrellevadas en su mayoría por las jóvenes. Esto último se vincula con ciertos roles domésticos que son transmitidos generacionalmente por sus progenitores, quienes les demandan mayores responsabilidades de cooperación a las mujeres y las obligan a quedarse en el hogar por su «seguridad», prohibiéndoles «salir a trabajar». Como nos relataba Camila, una joven de 22 años de barrio Toba: 
Mis hermanos se van a cirujear ${ }^{9}$ al centro, rebuscarse, no queda otra que salir así. Van más ellos los varones, porque las mujeres tendríamos que quedar en casa por la inseguridad que hay afuera, según lo que nos dicen ellos. Que en parte yo lo entiendo, que hay mucha inseguridad, más para una nena de 13 y 15 años llevarlas y buscar algo en la basura, y que otro degenerado venga, no. Eso es lo que les preocupa más a ellos, entonces nos dejan en la casa, mi mamá no nos deja. (Camila, joven de 22 años, barrio Toba, 16 de octubre de 2019).

De esta manera se va construyendo una «socialización diferencial» entre varones y mujeres vinculados con ciertos aprendizajes, roles y vivencias mediadas por la división sexual del trabajo doméstico al interior del hogar. Como ha sido planteado por diferentes investigadores, en los sectores de mayor vulnerabilidad y pobreza, estos imaginarios operan con fuerza diferenciando la distribución de responsabilidades respecto del trabajo doméstico y extradoméstico, e incidiendo, así, en la preferencia a emplearse en determinados empleos y no en otros (Millenaar y Jacinto, 2010).

Hemos registrado que este tipo de tareas reproductivas y de cuidado al interior del hogar son significadas como «ayuda». Si nos detenemos en la construcción de sentido sobre la noción de ayuda, observamos que se vincula con que la actividad desarrollada no es concebida como trabajo, sino que se enmarca dentro de las dinámicas y obligaciones familiares. Consideramos que esto está ligado con las construcciones hegemónicas sobre el trabajo doméstico, el cual tendió a ser despojado ideológicamente de su contenido de trabajo y de reproducción social, invisibilizando su importancia social para la sobrevivencia y el desarrollo de la sociedad capitalista (Peredo Beltrán, 2003; Narotzky, 2004).

\footnotetext{
${ }^{9}$ Cirujeo: palabra del lunfardo argentino. Se refiere al trabajo de recolección de residuos reciclables como actividad de subsistencia.
} 
A partir de este recorrido por las diferentes actividades, prácticas y significaciones que constituyen los modos de ganarse la vida de los y las jóvenes, observamos que estas incluyen empleos precarios, principalmente en los sectores de la construcción y comercio; actividades dentro del cuentapropismo comercial e informal; la participación en diferentes programas de dependencias estatales y organizaciones sociales y, para el caso de las mujeres jóvenes, variadas tareas reproductivas y de cuidado en el hogar. Estos recorridos y posibilidades laborales expresan ciertas desigualdades de género vinculadas tanto con las condiciones del mercado de trabajo y las redes informales presentes en los territorios donde habitan, como también por mandatos culturales asociados a los géneros y que inciden en la división de las responsabilidades domésticas y usos del tiempo entre varones y mujeres.

En lo que respecta a los sentidos sociales vinculados al trabajo, dimos cuenta de que la heterogeneización de sus formas genera una serie de jerarquías simbólicas, a partir de las cuales el trabajo asalariado, bajo dependencia y remunerado ocupa un lugar de prestigio, frente a las formas cuentapropistas y/o informales —asociadas al rebusque - y las domésticas — significadas como ayuda.

En el próximo apartado, veremos de qué manera esta diversidad de opciones y posibilidades ligadas al trabajo imprime particularidades a las acciones estatales que se tramitan cotidianamente en los dispositivos estatales territoriales.

\section{RESIGNIFICACIONES, TENSIONES Y ADAPTACIONES DEL TRABAJO EN LA GESTIÓN COTIDIANA DE LAS POLÍTICAS DE INCLUSIÓN LABORAL}

En el siguiente apartado nos interesa restituir algunas de las resignificaciones, tensiones y adaptaciones de las «políticas» y sus prescripciones 

de jóvenes que participan en programas de formación sociolaboral en contextos de pobreza urbana de la ciudad de Rosario, Argentina

normativas generadas por los trabajadores estatales y los jóvenes en su cotidianeidad laboral. Consideramos que estas son parte de procesos activos de reinterpretación y resignificación que se originan en la efectiva puesta en práctica de las políticas, donde se tramita una heterogeneidad de sentidos y prácticas de trabajo que imprimen particularidades a las acciones estatales sobre los y las jóvenes.

Para su análisis, tomaremos en consideración dos «unidades productivas» desarrolladas dentro del Programa Nexo Oportunidad: una se orienta a la fabricación de muebles de madera y otra al cultivo de plantas aromáticas para la elaboración de secados y cosmética natural para el propio consumo y/o comercialización. Estos espacios productivos funcionan tres veces por semana en las instalaciones del Centro Cultural «El Obrador» y son sostenidos por un capacitador en cada rubro productivo - contratados por el Ministerio de Educación de la provincia de Santa $\mathrm{Fe}$ - y dos «acompañantes» que cumplen tareas de registro, asistencia y retrabajo de problemáticas de los participantes contratados por el Ministerio de Desarrollo Social de la provincia de Santa Fe. Los grupos de jóvenes están compuestos por diez integrantes de entre 17 y 25 años en cada capacitación: tres mujeres y siete varones en carpintería, y nueve mujeres y un varón en el espacio de huerta.

Entre las principales actividades realizadas en el marco de las unidades productivas identificamos: la adquisición de algunos saberes y prácticas vinculados a los oficios — carpintería y huerta_, la venta de las producciones que se fueran realizando - ya sea por encargo o en las ferias del barrio-y el trabajo en diferentes problemáticas presentadas por los y las jóvenes. Asimismo, se planifican actividades que potencian los aprendizajes a partir de visitas a encuentros, charlas y museos vinculados con los rubros productivos, intercambios con otros grupos de jóvenes, capacitaciones paralelas en marketing y diseño, entre otras. Además de las actividades propias del espacio de 
capacitación, se incluía un espacio de merienda y almuerzo; salidas grupales a parques públicos, cines y otras excursiones; celebraciones del día del niño; y las movilizaciones de protesta organizadas por los trabajadores estatales y organizaciones sociales, quienes sostenían las actividades del Programa.

La mayor parte de estas actividades son impulsadas por los y las trabajadores estatales del territorio, quienes nos relataban que su labor resulta «artesanal» en tanto requiere de intervenciones de carácter personalizado y creativo y por «fuera de los protocolos de acción». Es importante destacar que, en paralelo al sostenimiento de estas actividades, los trabajadores cuestionaban las disposiciones explícitas y denunciaban un conjunto de problemas ${ }^{10}$, entre los que sobresalen la escasez y precariedad del personal estatal, la carencia de recursos materiales y presupuestarios para sostener sus actividades y el trabajo con la «emergencia» que demandan los jóvenes y que desbordan sus posibilidades de resolución.

Ahora bien, en cuanto a los modos de ingreso a las capacitaciones y unidades productivas de los y las jóvenes, pudimos identificar que llegaban generalmente a través de conocidos, amigos y/o familiares. Determinamos que, de manera recurrente en las entrevistas, su acercamiento se vincula con la posibilidad de «hacer algo» o «no estar al pedo», lo que se vincula con la irregularidad e inestabilidad que caracteriza la oferta de actividades y oportunidades laborales que señalábamos en el apartado anterior. Otros de los sentidos otorgados a la participación en los dispositivos estatales se vinculan con la posibilidad de generar un ingreso que les permita «ayudar» a sus familias en

10 Durante nuestro trabajo de campo participamos de numerosas reuniones y movilizaciones orientadas al fortalecimiento del Programa «Nueva Oportunidad», donde sus trabajadores denunciaban: precariedad laboral y la necesidad de cargos de planta permanente, así como mayor presupuesto para sus actividades. La organización de estas actividades estaba a cargo de referentes de organizaciones sociales que gestionaban estas capacitaciones y trabajadores estatales nucleados en AMSAFE (Asociación del Magisterio de Santa Fe), que es el sindicato en el que se organizaron capacitadores de los cursos de oficios. 
la reproducción del hogar. Para estos participantes, «hacer una capacitación»e intentar «armar un emprendimiento» está en concordancia con las expectativas de generar ingresos dinerarios, «sentirse útiles»y, de esta manera, afrontar las incertidumbres y fluctuaciones laborales que atraviesan. Como destacábamos anteriormente, los recorridos sociolaborales dentro del trabajo remunerado están signados generalmente por su precariedad e inestabilidad. En efecto, es recurrente que algunos participantes sostengan la participación en los momentos en que no tenían trabajo o para complementar los ingresos que generaban en simultáneo con otras actividades laborales no asalariadas.

Es importante destacar que el sostenimiento de la actividad apareció en algunos relatos de los varones como posibilidad de «rescate», de no «estar en la mala». Bajo esta categoría social, refieren a que el sostenimiento de la capacitación les permite tomar distancia de diferentes experiencias relacionadas con el consumo problemático de sustancias y/o situaciones delictivas. En tal sentido, relevamos que, de manera frecuente, eran los padres quienes «obligaban»a sus hijos a sostener la participación en estos espacios, con la expectativa de la «superación» o «salida» de las situaciones de peligrosidad que afrontaban los jóvenes. Asimismo, registramos que esta puede articularse con otras estrategias para salir «de la mala», que está vinculada con el alejamiento del entorno barrial y de los amigos, a partir de su migración o traslado para trabajar con parientes que residen en otros lugares. Desde la perspectiva de los adultos, salir del barrio e irse a trabajar y/o estudiar resulta una posibilidad para que los jóvenes se «rescaten» de las situaciones riesgosas que enfrentan en el barrio. Por otro lado, identificamos que los lugares de recepción y los vínculos que allí se entablan se vuelven importantes para la inserción laboral y la adquisición de ciertos conocimientos en diferentes oficios.

Durante el dictado de los cursos de capacitación y producción la asistencia de la mayoría de los y las jóvenes era fluctuante, dando lugar a 
discusiones, preocupaciones y una cierta insistencia de los trabajadores que realizaban llamados o visitas a sus domicilios para contactarlos, convocarlos a asistir o preguntarles por los motivos de sus ausencias.

Durante nuestras observaciones participantes en los espacios de trabajo, identificamos que la dinámica grupal se da en el marco de gran confianza y «calidez» entre los participantes donde se debatían diferentes situaciones que traían los y las jóvenes y que demandaban otro tipo de tareas. Nos referimos a situaciones tales como la violencia institucional, de género, problemáticas de salud o habitacional, para las cuales los trabajadores solían realizar actividades de debate, reuniones con otras instituciones y/o gestiones para coordinar turnos de consultas en los Centros de Salud o la realización de trámites administrativos.

En cuanto a la especificidad del trabajo de capacitación y producción, distinguimos que se desarrollan como talleres eminentemente prácticos, en los que se articula el tratamiento de determinados contenidos específicos del aprendizaje del rubro con el avance en la elaboración de diferentes productos para su posterior comercialización. La transmisión de conocimientos se realiza mirando y haciendo a la par del capacitador, quien explica y ejecuta detalladamente diferentes tareas $\mathrm{y}$, posteriormente, los jóvenes realizan las tareas bajo el control de los capacitadores.

El aprendizaje del oficio se acompasa con la necesidad de sostener un ritmo de trabajo para que no se "retrasen con los pedidos". Esto se hacía evidente en las discusiones entre participantes que resaltaban que "acá se viene a trabajar" $\mathrm{y}$, por lo tanto, se enojaban con quienes trabajaban despacio o perdían el tiempo comiendo o sin hacer nada. Identificamos que estas tensiones se generan entre quienes tienen mayores experiencias dentro de trabajos formales y quienes no, ya que los primeros son quienes demandan mayor compromiso a estos últimos. Generalmente, frente estos conflictos, los capacitadores y acompañantes 
resaltaban la importancia del trabajo colectivo y compañerismo, respetando diferencias entre los participantes.

En algunos talleres, la discusión sobre el oficio y el trabajo grupal se entrelazaba con comentarios sobre las tareas específicas de la gestión del «emprendimiento», entre las que podemos mencionar: la confección de presupuesto de trabajo, la importancia de dominar el lenguaje técnico, las modalidades de comercialización y el trato de los posibles compradores, el uso de redes de comunicación virtuales, la administración del dinero, entre otras. En estas instancias se recuperaban saberes y experiencias previos de los y las jóvenes, quienes participaban activamente de estas tareas.

En lo que respecta a la comercialización de las producciones realizadas se destaca la recuperación de ámbitos y dinámicas barriales y familiares de los y las jóvenes. Nos referimos a que, entre los «espacios de venta», se incluyen las ferias populares en las cuales ya participan con sus familias. La asistencia a estos espacios puede realizarse tanto durante los días de «taller» como los fines de semana - bajo la responsabilidad de los y las jóvenes, acompañados por los trabajadores estatales - Asimismo, se recuperan estrategias de venta que los jóvenes realizaban con anterioridad, como son las denominadas "rifas". Estas suelen organizarse en fechas específicas (fin de año) y se realizan dividiendo una cantidad de números por cada joven. Los y las jóvenes comentan que realizan recorridas por el barrio, en la escuela y con sus familiares para la venta de las mismas. Para su sorteo, se organizaban eventos presenciales para "hacerse conocer" en el barrio. Esto muestra cómo las relaciones de cercanía (vecinales y/o familiares) son las más relevantes para circular y comercializar los productos.

Por otro lado, también registramos que la dinámica de «pedidos» y «encargos» se realiza recuperando los vínculos y redes entre las distintas instituciones estatales o sociales al interior del barrio. Nos referimos a que 
muchos de estos eran «pedidos» eran realizados por trabajadores de los Centros de Salud, clubes barriales y/o organizaciones territoriales.

Es importante destacar que en los procesos de producción $y$ comercialización, la división de las faenas no sigue patrones fijos: varones y mujeres realizan indistintamente diversas ocupaciones (no relevamos tareas «femeninas» o «masculinas»). En relación con los contenidos de la capacitación, todos los jóvenes destacan que a partir del taller lograron conocer el oficio y creen que podrían desarrollar la actividad por fuera del espacio.

En relación con la administración y uso del dinero generado, registramos que esto es tomado por los capacitadores y/o acompañantes, quienes recolectan y guardan el dinero para su posterior división en tanto garantía de equidad y seguridad entre los mismos. Esto fue notorio en varias oportunidades en las que los jóvenes reclamaban el dinero para «alcanzárselo» a algún participante que no estaba presente y los trabajadores recordaban que la norma de división era la «planta en mano». Esto se vincula con lo que los trabajadores enuncian como la «urgencia» material y necesidad de «plata ya», a partir de la cual, para los trabajadores se vuelve difícil la administración de los propios jóvenes.

Por otro lado, los y las jóvenes diferencian esta actividad de sus experiencias laborales en tanto la misma les «gusta», demanda menos «tiempo» y se «aprende», también les permite ampliar sus expectativas laborales. En tal sentido, su carga horaria y la posibilidad de que se realice al interior del barrio les permiten la continuidad y complementariedad con las tareas de cuidado de hijos, hermanos, etc. Esto tiene particular importancia para el caso de las participantes que tiene hijos pequeños o hermanos menores a cargo, quienes, como describimos en el apartado anterior, son las que asumen las tareas reproductivas y de cuidado al interior del hogar. De esta manera, el sostenimiento de las capacitaciones se erige como posibilidad de «conciliar» la vida laboral y 

de jóvenes que participan en programas de formación sociolaboral en contextos de pobreza urbana de la ciudad de Rosario, Argentina

el proyecto de la maternidad, las responsabilidades y obligaciones en el cuidado de sus hijos, que recaían generalmente sobre ellas.

Otro elemento que se destacó en las entrevistas con los y las jóvenes refiere a los vínculos afectivos generados con los trabajadores a cargo del taller, los cuales son valorados positivamente y generalmente son los que les permiten continuar más allá de dichas fluctuaciones temporales. Asimismo, se destacan los lazos generados entre los jóvenes, ya que señalan que han podido armar un grupo en donde «se llevan bien», hay «confianza» y «responsabilidad» grupal. Resulta importante mencionar que algunos jóvenes se proyectan dentro de las actividades desarrolladas en estos espacios y lo piensan como una posibilidad laboral para su futuro.

\section{REFLEXIONES FINALES}

En este trabajo nos propusimos analizar los modos de ganarse la vida de jóvenes que participan de políticas de inclusión laboral y cómo estos se interpenetran en los procesos de implementación cotidiana en contextos de pobreza urbana. A partir del mismo pudimos iluminar el carácter complejo, activo y contextual de la gestión cotidiana del trabajo en dispositivos estatales, en sus múltiples resignificaciones y apropiaciones locales.

En lo que respecta al programa seleccionado para nuestro análisis, identificamos que se parte del diagnóstico de la lejanía de los y las jóvenes con las instituciones laborales y, por ende, de la «cultura del trabajo». De esta manera, entre sus lineamientos y modalidades de la intervención se propone un abordaje «integral», orientado a incidir sobre las carencias «formativas»y «disposiciones» hacia el trabajo, como también en los «proyectos de vida» de los y las jóvenes. Asimismo, adquiere centralidad lograr una «proximidad territorial» con la población destinataria, localizando a los equipos de trabajo en instituciones 
estatales descentralizadas o instituciones de la sociedad civil situados en los contextos sociourbanos donde habitan los jóvenes. Entre las acciones previstas se incluyen diferentes trayectos de capacitación y formación laboral en diferentes sectores y rubros de actividad, la inserción de los jóvenes en pasantías en empresas y la conformación de unidades productivas (emprendimientos).

Consideramos que estos diagnósticos y supuestos estatales son construidos sobre la base de concepciones que reducen el trabajo al trabajo asalariado formal (y, por lo tanto, a lo que no se accede, o se accede en menor medida). De esta manera, se tiende a invisibilizar las divergentes vinculaciones que estos sujetos tienen con el mundo laboral dentro del universo de posibilidades presentes en los territorios analizados. En tal sentido, a partir de nuestro acercamiento a los modos de ganarse la vida de los y las jóvenes, registramos que muchos participantes tenían amplias trayectorias laborales asalariadas atravesadas por una gran precariedad y por una vasta irregularidad temporal. El acceso a estos trabajos generalmente está mediado por las redes vecinales y familiares y se desarrollan por fuera de los asentamientos. Asimismo, relevamos que desarrollan una variedad de prácticas y redes de trabajo no salariales que incluyen formas de trabajo cuentapropista, principalmente: en el comercio, bajo la forma de vendedores ambulantes y callejeros de toda clase de artículos y baratijas; prácticas vinculadas con la recolección de materiales reciclables, desechados en el espacio urbano, para su venta y/o uso doméstico. También evidenciamos circuitos laborales vinculados a la participación en diferentes programas de dependencias estatales y organizaciones sociales y políticas del territorio. Por último, una serie de actividades reproductivas y de cuidado al interior del hogar, las cuales generalmente están a cargo de las jóvenes. En cuanto a las significaciones construidas sobre estas prácticas y actividades laborales, identificamos que más allá de la precariedad en la que se desarrolla el trabajo asalariado, este es significado como «verdadero» trabajo frente a las opciones más informales, que son significadas como «rebusque» y/o frente al trabajo 

de jóvenes que participan en programas de formación sociolaboral en contextos de pobreza urbana de la ciudad de Rosario, Argentina

reproductivo y doméstico como «ayuda». Sin embargo, esto no niega sentidos negativos, en relación con los contenidos y formas concretos en los que se lo ejerce, a saber, las arbitrariedades patronales, el desgaste físico y las malas remuneraciones.

Estas heterogéneas experiencias y relaciones que los y las jóvenes entablan con el trabajo (precario, informal, reproductivo, de cuidado) son tensionadas en la propia puesta en acto de las políticas, a partir de la generación de consensos y apropiaciones recíprocas entre los distintos actores que participan de su gestión cotidiana en el ámbito barrial/territorial.

Entre los principales hallazgos identificamos que, entre las motivaciones y posibilidades de sostenimiento de la experiencia, los y las jóvenes ubican que su acercamiento se relaciona con las escasas y fluctuantes posibilidades de acceso al trabajo remunerado, permitiéndoles generar un ingreso dinerario. Asimismo, aparece como posibilidad de «rescatarse» frente a situaciones de peligrosidad que afrontan vinculados principalmente con el consumo problemático y/o actividades consideradas «delictivas». Estas cuestiones tuvieron mayor gravitación en los relatos de los varones. Por el contrario, entre las mujeres apareció vinculado con la posibilidad de «conciliar» la vida laboral y familiar, en tanto su carga horaria y cercanía al interior del barrio les permite sostener el cuidado de los hijos. De esta manera, observamos cómo, para el caso femenino, las tareas reproductivas resultan estar bajo su responsabilidad.

En lo que respecta a la dinámica de trabajo, se destaca cómo la aprensión del oficio se realiza en la propia puesta en acto de la producción y a la par del tratamiento de diferentes problemáticas que son planteadas por los y las jóvenes. En tal sentido, hemos relevado que valoran positivamente de la experiencia: los vínculos de afecto construidos entre los participantes y trabajadores estatales, el aprendizaje del oficio y la organización colectiva del trabajo. De esta manera, la participación es significada como lo que les «gusta» y con sentidos dicotómicos 
y opuestos a otras experiencias laborales, principalmente bajo la forma asalariada.

En relación con la comercialización de las producciones realizadas se destaca la recuperación de ámbitos y dinámicas barriales y familiares, a partir de la participación en las ferias populares de los asentamientos y a través de vínculos con vecinos y familiares. Por otro lado, hemos identificado limitaciones en torno a la repartición de los ingresos dinerarios obtenidos. Estas tareas suelen recaer en los trabajadores estatales del territorio, quienes aparecen como garantía de la repartición equitativa.

A partir de este recorrido, podemos plantear que en la dinámica de trabajo generada cotidianamente se reconocen diferentes actividades laborales que realizan los jóvenes que permean la gestión cotidiana del programa. De esta manera, se amplía el universo de trabajo, se redefinen las actividades y tareas cotidianas y se generan instancias de producción y comercialización que recuperan las propias dinámicas territoriales y familiares.

ENVIADO: 6 DE ABRIL DE 2021 ACEPTADO: 26 DE MAYO DE 2021 


\section{BIBLIOGRAFÍA}

AssusA, G. (2014). Dispuestos a trabajar. Aproximación a la visión de los agentes estatales de un programa de empleo para jóvenes en Argentina. Revista de Antropología Social, 23, 181-208. https://www.redalyc.org/articulo.oa?id=83832495009

AssusA, G. y BRANDÁN ZEHNDER, M. G. (2014). La empleabilidad, sus sentidos y dispositivos: un estudio de caso desde la perspectiva de los beneficiarios del Programa Jóvenes Más y Mejor Trabajo en Córdoba Capital, Argentina. $\begin{array}{llll}\text { Sociedade } \quad \text { Cultura, } & 17, & 121-132 .\end{array}$ https://www.redalyc.org/articulo.oa?id=703/70340850010

Balardini, S. (1999). Políticas de juventud: conceptos y la experiencia Argentina. Última Década, $10, \quad$ 89-128. https://www.redalyc.org/pdf/195/19501004.pdf

Bourgois, P. (2010). En busca de respeto. Vendiendo crack en Harlem. Buenos Aires: Siglo XXI.

Brown, B. (2017). Sistema de Protección social y Programas de Transferencias Monetarias Condicionadas. El "paradigma de activación» en Argentina 2003-2013. Tesis Maestría, Facultad de Ciencias Sociales, Universidad de Buenos Aires, Argentina.

Castagna, A. y Véntola, V. (2015). Análisis de la Inserción laboral de los Jóvenes en el Aglomerado Gran Rosario en la última década. En IX Jornadas de Ciencia y Tecnología de la Universidad Nacional de Rosario, Argentina.

CORTÉS, R. y KESSLER, G. (2013). Políticas, ideas y expertos en la cuestión social de la Argentina democrática (1983-2012). Revista de Indias, 73(257), 239264. DOI: $10.3989 /$ revindias.2013.010

De Ibarrola, M. (2002). Hacia una mirada integral de la formación de los jóvenes para el trabajo. En Desarrollo local y formación: Hacia una mirada integral de la formación de los jóvenes para el trabajo (pp. 15-38). Montevideo: Cinterfor.

FERNÁNDEZ ÁlvAREZ, M. I. (2016). Experiencias de precariedad, creación de derechos y producción colectiva de bienestar(es) desde la economía popular. Revista Ensambles en sociedad, política y cultura, 4/5, 72-89. http://www.revistaensambles.com.ar/ojs-

2.4.1/index.php/ensambles/article/view/76/50

IsACOVICH, P. (2016). De las políticas orientadas a jóvenes pobres a la producción social de la vida y las políticas en un barrio popular. Una etnografía desde el sur de la Ciudad de Buenos Aires. IX Jornadas de Sociología de la UNLP, 5 al 7 de diciembre de 2016, Ensenada, Argentina. En 
Memoria

Académica.

http://www.memoria.fahce.unlp.edu.ar/trab_eventos/ev.8928/ev.8928.pdf . (2013). Hacer el Estado, regular la vida: una etnografía de políticas de

juventud. Avá, 22 , 33-56. http://argos.fhycs.unam.edu.ar/handle/123456789/611

JACINTO, C. (2016). Redefiniendo la empleabilidad en los programas de formación e inserción laboral de jóvenes. El rol de las organizaciones de la sociedad civil. En E. FAUR (comp.), Repensar la inclusión social. Políticas públicas y sociedad civil en la Argentina (1991-2016) (pp. 109-134). Buenos Aires: Fundación Tzedaká.

. (2010). Veinte años de políticas de formación para el empleo de jóvenes vulnerables en América Latina: persistencias y reformulaciones. En La construcción social de las trayectorias laborales de jóvenes: políticas, instituciones, dispositivos y subjetividades (pp. 119-148). Buenos Aires: Teseo, Ides.

- (2008). Los dispositivos recientes de empleo juvenil: institucionalidades, articulaciones con la educación formal y socialización laboral. Revista de Trabajo, 6, 123-142. http://www.derechoshumanos.unlp.edu.ar/assets/files/documentos/losdispositivos-recientes-de-empleo-juvenil-institucionalidades-articulacionescon-la-educacion-formal.pdf

. (2002). Los jóvenes, la educación y el trabajo en América Latina. Nuevos temas, debates y dilemas. En Desarrollo local y formación: Hacia una mirada integral de la formación de los jóvenes para el trabajo (pp. 67102). Montevideo: Cinterfor.

KRAUSKOPF, D. (2004). Perspectivas sobre la condición juvenil y su inclusión en las políticas públicas. En E. Gerber y S. BALARDInI (comps.), Políticas de juventud en Latinoamérica. Argentina en perspectiva (pp. 12-24). Buenos Aires: Fundación Friedrich Ebert.

MANZANO V. (2013). La politica en movimiento. Movilizaciones colectivas y políticas estatales en la vida del Gran Buenos Aires. Rosario: Prohistoria Ediciones.

MARTICORENA, C. (2014). Trabajo y negociación colectiva. Los trabajadores en la industria argentina, de los noventa a la postconvertibilidad. Buenos Aires: Imago Mundi.

MenÉnDEZ, E. (2010). La parte negada de la cultura. Rosario: Prohistoria Ediciones.

. (1999). Trabajo y significación subjetiva, continuidad cultural, determinación económica y negatividad. En V. NOVELo (comp.), Historia y cultura obrera (pp. 250-279). Ciudad de México: CIESAS. 

de jóvenes que participan en programas de formación sociolaboral en contextos de pobreza urbana de la ciudad de Rosario, Argentina

MillenaAR, V. (2012). Incidencias de la capacitación para el trabajo en las trayectorias de mujeres jóvenes de bajos recursos. Tesis de Maestría en Ciencias Sociales, Buenos Aires, UNGS-IDES.

MillenaAR, V. y Jacinto, C. (2010). La incidencia de los dispositivos en la trayectoria laboral de los jóvenes. Entre la reproducción social y la creación de oportunidades. En C. JACINTO (coord.), La construcción social de las trayectorias laborales de jóvenes. Políticas, instituciones, dispositivos y subjetividades (pp. 181-224). Buenos Aires: Teseo.

Muñiz Terra, L., Roberti, E., Deleo, C. y Hasicic, C. (2013). Trayectorias laborales en Argentina: una revisión de estudios cualitativos sobre mujeres y jóvenes. Revista Lavboratorio, 14(25), 57-79.

Navarrete, E., Padrón Innamorato, M. y Silva Arias, A. (2012). La inserción laboral de los jóvenes y las políticas de empleo en Colombia, México y Uruguay. En M. PADrón InNAMORATO y L. GANDINI (coords.), Población y trabajo en América Latina: abordajes teóricoconceptuales y tendencias empíricas recientes. Serie investigaciones $\mathrm{N}^{\circ}$ 14. Asociación Latinoamericana de Población (ALAP)-Fondo de Población de las Naciones Unidas (UNFPA).

NArotzky, S. y Besnier, N. (2014). Crisis, Value, and Hope: Rethinking the Economy. Current Anthropology, 55(S9), 4-16. DOI: 10.1086/676327. https://www.jstor.org/stable/10.1086/676327

. (2013). Economías ordinarias: valores escondidos. Otra antropología de la crisis desde el sur de Europa. Antrópica. Revista de Ciencias Sociales y Humanidades, 1(2), 67- 76. http://www.antropologia.uady.mx/revista/antropica/num02/2_3_Narotsky.pd f

. (2004). Antropología económica. Nuevas tendencias. Barcelona: Melusina.

Peredo Beltrán, E. (2003). Mujeres, trabajo doméstico y relaciones de género: reflexiones a propósito de la lucha de las trabajadoras bolivianas. en Mujeres y trabajo: cambios impostergables (pp. 54-65). Porto Alegre: Veraz Comunicação.

PÉREZ, P. (2013). Empleabilidad, motivación por trabajar y políticas de empleo para jóvenes en Argentina. Cuestiones de Sociología, 9, 89-92. http://www.memoria.fahce.unlp.edu.ar/art_revistas/pr.5893/pr.5893.pdf

. (2010). La inserción laboral de jóvenes urbanos en la Argentina postConvertibilidad. Revista Atlántida, 2, 73-93. https://pdfs.semanticscholar.org/7cb0/6cc52a5c8ec09ee645d672053b23d376 fdeb.pdf 
SALVIA, A. (2010). Heterogeneidad estructural y segmentación del mercado de trabajo en dos contextos económicos. Argentina 1998-2006. VI Congreso de la Asociación Latinoamericana de Sociología del Trabajo, Ciudad de México.

SÁNCHEZ, S. (2006). Juventud y conformación de identidad. Modos de grupalidad juvenil en ámbitos de pobreza y diversidad sociocultural. Tesis de grado inédita. Facultad de Humanidades y Artes, Universidad Nacional de Rosario, Argentina.

SHAPIRO, J. (2000). Educación y desempleo. La demanda de mano de obra en el Gran Rosario. En E. LIBIA, Escuela y ciudad. Exploraciones de la vida urbana (pp. 44-50). Rosario: UNR Editora.

VALVERDE VIESCA, K. (2012). Reforma y políticas juveniles en México: avances y retrocesos institucionales. Journal de Ciencias Sociales. Universidad de Quilmes, 1(1), 42-61.

VÁzQUEZ, M. (2019). Reconfiguraciones de las élites estatales y de las producciones socioestatales de juventudes en Argentina (2015-2019). Revista Perfiles Latinoamericanos, 28(55), 55-81.

Vitali, S. (2018). Políticas sociales, trabajo y pobreza urbana. Análisis de la gestión cotidiana del trabajo en políticas socio-laborales implementadas por la Secretaría de Economía Social en dos asentamientos irregulares de la ciudad de Rosario (Santa Fe). Revista de la Escuela de Antropología, XXIV, 1-18.

\section{FUENTES}

Informe ABRE. ODSA/UCA-Gobierno de la Provincia de Santa Fe.

Ordenanza Municipal No 684/16: Programa Nueva Oportunidad

La Nación, 11 de abril de 2014.

El Ciudadano, 2 de noviembre de 2018. 